\title{
An On-Chip, Multichannel Droplet Sorter Using Standing Surface Acoustic Waves
}

\author{
Sixing Li, ${ }^{\dagger, \dagger}$ Xiaoyun Ding, ${ }^{\dagger}$ Feng Guo, ${ }^{\dagger}$ Yuchao Chen, ${ }^{\dagger}$ Michael Ian Lapsley, ${ }^{\dagger}$ Sz-Chin Steven Lin, ${ }^{\dagger}$ \\ Lin Wang, J. Philip McCoy," Craig E. Cameron, ${ }^{\S}, \perp$ and Tony Jun Huang, ${ }^{*}, \neq$
}

\author{
${ }^{\dagger}$ Department of Engineering Science and Mechanics, The Pennsylvania State University, University Park, Pennsylvania 16802, United \\ States \\ ${ }^{\ddagger}$ Cell and Developmental Biology (CDB) Graduate Program, The Huck Institutes of the Life Sciences, The Pennsylvania State \\ University, University Park, Pennsylvania 16802, United States \\ ${ }^{\S}$ Ascent Bio-Nano Technologies Inc., State College, Pennsylvania 16801, United States \\ ${ }$ National Heart, Lung, and Blood Institute at NIH, Bethesda, Maryland 20892, United States \\ ${ }^{\perp}$ Department of Biochemistry and Molecular Biology, The Pennsylvania State University, University Park, Pennsylvania 16802, \\ United States
}

\section{Supporting Information}

ABSTRACT: The emerging field of droplet microfluidics requires effective on-chip handling and sorting of droplets. In this work, we demonstrate a microfluidic device that is capable of sorting picoliter water-in-oil droplets into multiple outputs using standing surface acoustic waves (SSAW). This device integrates a single-layer microfluidic channel with interdigital transducers (IDTs) to achieve on-chip droplet generation and sorting. Within the SSAW field, water-in-oil droplets experience an acoustic radiation force and are pushed toward the acoustic pressure node. As a result, by tuning the frequency

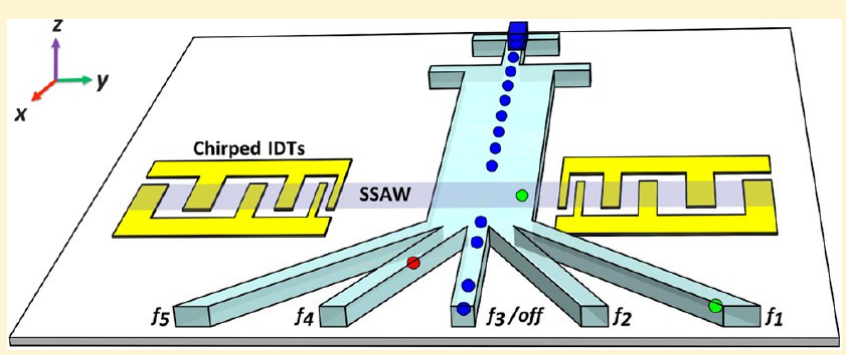
of the SSAW excitation, the position of the pressure nodes can be changed and droplets can be sorted to different outlets at rates up to 222 droplets $\mathrm{s}^{-1}$. With its advantages in simplicity, controllability, versatility, noninvasiveness, and capability to be integrated with other on-chip components such as droplet manipulation and optical detection units, the technique presented here could be valuable for the development of droplet-based micro total analysis systems ( $\mu$ TAS).

$\mathrm{I}^{\mathrm{n}}$ $\mathrm{n}$ recent years, droplet-based microfluidics ${ }^{1,2}$ has demonstrated tremendous potential in many chemical and biological assays due to its advantages of low sample consumption, high throughput, short mixing time, elimination of cross contamination, and capacity to be integrated with other lab-on-a-chip devices. ${ }^{3-8}$ In particular, the high throughput inherent in droplet microfluidics makes it extremely promising for various chemical or biological screenings. ${ }^{9-15}$ Droplet-based screening largely relies on precise isolation of individual droplets of interest from other droplets; thus, it is essential to develop effective, on-chip droplet-sorting techniques. Passive sorting methods such as hydrodynamic self-sorting ${ }^{16}$ and deterministic lateral displacement-based sorting ${ }^{17}$ make use of the size difference between droplets, but they suffer from an incapability to sort droplets with similar sizes. Meanwhile, many active droplet-sorting techniques have been developed in recent years. ${ }^{18-33}$ Among the existing micro object manipulation techniques, acoustic-based approaches offer significant advantages in compactness, versatility, and noninvasiveness. Recently, low-power acoustic waves have been extensively utilized in manipulation of micro objects (e.g., bubble, particle, cell, organism, and droplet) due to their harmless nature to biological objects. ${ }^{32-59}$ In our previous studies, we have used standing surface acoustic waves (SSAW) to achieve focusing, separation, sorting, and patterning of microparticles and cells in microfluidics. ${ }^{60-66}$

In this work, we report a SSAW-based, on-chip, multichannel droplet-sorting technique that takes advantages of the tunability and control ${ }^{65,66}$ offered by chirped interdigital transducers (IDTs). Our SSAW-based approach is fundamentally different from the recently demonstrated traveling surface acoustic wave (TSAW)-based droplet-sorting technique: ${ }^{33}$ our SSAW-based droplet-sorting method manipulates the objects (i.e., droplets) directly by the acoustic radiation force, while the TSAW-based method actuates the fluid (i.e., oil) that surrounds the objects through the acoustic streaming effect. Our method has excellent controllability and a large range of translation, which renders it capable of precisely sorting cells into a great number (e.g., five) of outlet channels in a single step. This is a major advantage over most existing droplet-sorting methods, which typically

Received: February 20, 2013

Accepted: May 6, 2013

Published: May 6, 2013 

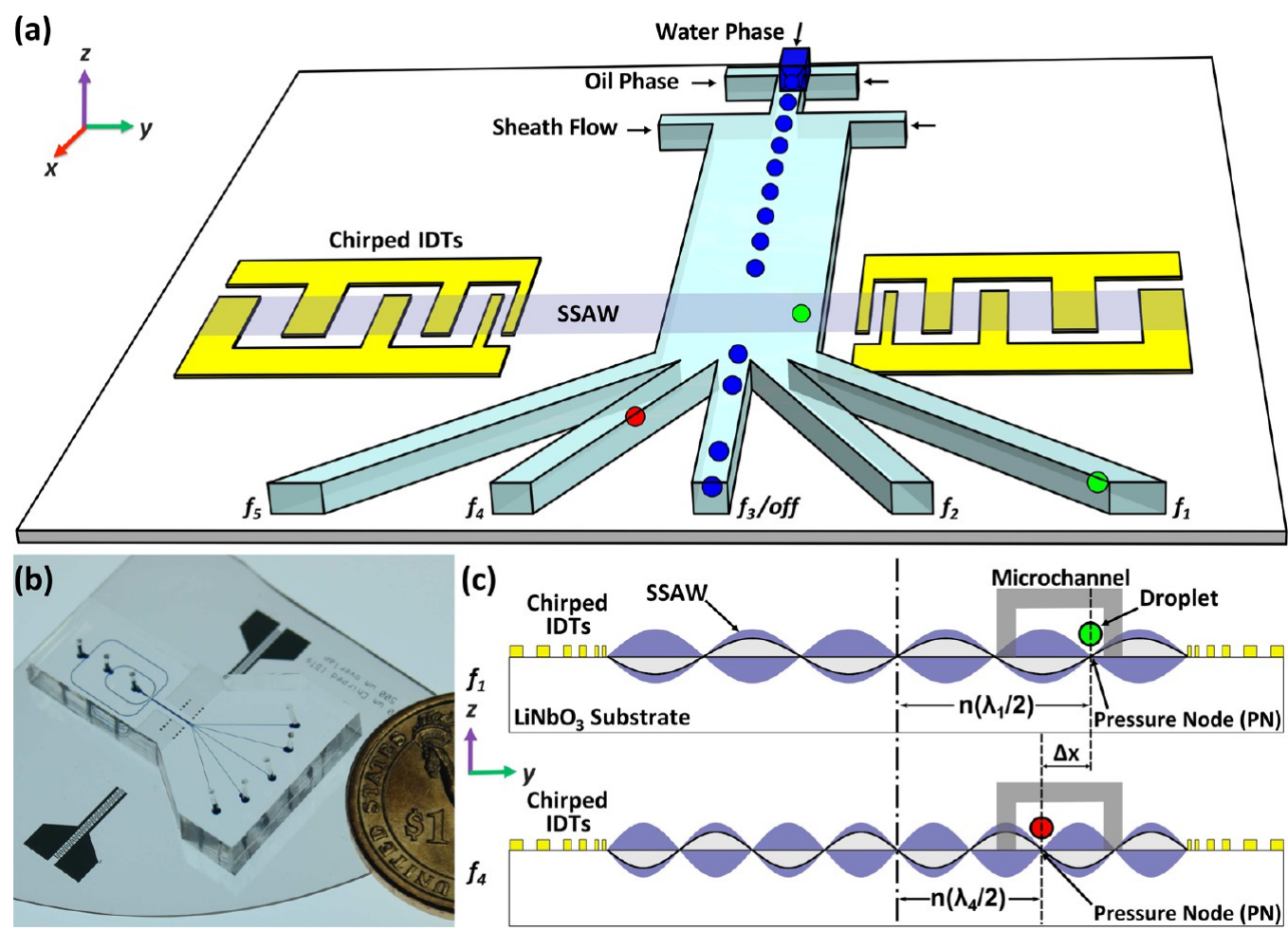

Figure 1. (a) Schematic of the SSAW-based multichannel droplet sorter. The shaded area indicates the SSAW field. (b) Optical image of our SSAWbased multichannel droplet sorter. (c) Cross-sectional view showing the SSAW-based, multichannel droplet-sorting mechanism.

only sort droplets into two outlet channels. Our approach is simple, versatile, and compact. We expect that it can be valuable in many chemical or biological analytical processes to facilitate the development of micro total analysis systems ( $\mu$ TAS).$^{67-74}$

\section{WORKING MECHANISM}

The integrated droplet-sorting device, shown in Figure 1a, consists of a flow-focusing, droplet-generation component ${ }^{2}$ and a SSAW-based, droplet-sorting component. The channel geometry was formed via standard soft lithography. ${ }^{75}$ In all of our devices, the height of the polydimethylsiloxane (PDMS) microchannel was $40 \mu \mathrm{m}$. The widths of the water-phase inlet, two oil-phase inlets, and two sheath-flow inlets were $50 \mu \mathrm{m}, 50$ $\mu \mathrm{m}$, and $100 \mu \mathrm{m}$, respectively. The widths of the main channel and the five outlet channels were 200 and $45 \mu \mathrm{m}$, respectively. The two oil sheath flows were used to control the interdroplet spacing. As shown in Figure 1a, the PDMS microchannel and a pair of narrow chirped IDTs (with a period of 360-400 $\mu \mathrm{m}$ and aperture of 300 or $500 \mu \mathrm{m}$ ) were aligned in parallel. Chirped IDTs are a variation of regular interdigital transducers (IDTs) which contain a linear gradient in their finger period. ${ }^{65,66}$ As a result, the wavelength of the excited surface acoustic waves (SAWs) is dependent upon the input frequency of the RF signal, while the amplitude of the SAWs is controlled by input voltage. The use of chirped IDTs rather than regular IDTs in our experiment is due to their capability to change the pressure node distribution of the generated SSAW field by tuning the input frequency. This is the basis for sorting droplets into multiple outputs.

The acoustic radiation force in the SSAW field was utilized to sort droplets. The primary acoustic radiation force acting on any microsphere in a SSAW field can be expressed as ${ }^{76}$

$$
\begin{aligned}
& F_{\mathrm{r}}=-\left(\frac{\pi p_{0}^{2} \mathrm{~V}_{\mathrm{c}}}{2 \lambda}\right) \phi(\beta, \rho) \sin (2 k x) \\
& \phi(\beta, \rho)=\frac{5 \rho_{\mathrm{c}}-2 \rho_{\mathrm{w}}}{2 \rho_{\mathrm{c}}+\rho_{\mathrm{w}}}-\frac{\beta_{\mathrm{c}}}{\beta_{\mathrm{w}}}
\end{aligned}
$$

where $p_{0}, \lambda, V_{\mathcal{c}} \rho_{\mathcal{c}} \rho_{\mathrm{w}}, \beta_{\mathcal{c}}$ and $\beta_{\mathrm{w}}$ are the acoustic pressure, acoustic wavelength, volume of the sphere, density of the sphere, density of the fluid, compressibility of the sphere, and compressibility of the fluid, respectively. In the equations, $\phi(\beta, \rho)$ describes the acoustic contrast factor of the sphere and determines whether the spheres move to pressure nodes or antinodes. In our work, the sizes of the water droplets range from 40 to $50 \mu \mathrm{m}$ in diameter, which are less than the wavelength of the SAWs $(360-400 \mu \mathrm{m})$, so the droplet can be approximated as a microsphere.

The mechanism of multichannel droplet sorting is illustrated in Figure 1c. When the chirped IDTs are excited at two different frequencies $\left(f_{1}\right.$ and $\left.f_{4}\right)$, the SSAW with wavelengths of $\lambda_{1} / 2$ and $\lambda_{4} / 2(\lambda=c / f$, where $c$ is the SAW propagation velocity on the $\mathrm{LiNbO}_{3}$ substrate) are formed, respectively. Since the microchannel is intentionally positioned closer to one chirped IDT than the other, the pressure node located inside the microchannel is the $n$th node from the centerline of the two IDTs, and the absolute distances between the node and the centerline at these two excitation frequencies are $n\left(\lambda_{1} / 2\right)$ and $n\left(\lambda_{4} / 2\right)$, respectively. Therefore, the translation of the droplet $(\Delta x)$ at the pressure node can be described as $\Delta x=n\left(\lambda_{1}-\right.$ $\left.\lambda_{4}\right) / 2=n\left(c / f_{1}-c / f_{4}\right) / 2$ for a frequency change from $f_{1}$ to $f_{4}$. As a result, droplets exit through different outlets as shown in Figure 1a. Therefore, our SSAW-based droplet sorter is capable of precise and controllable sorting of droplets to different outlets through the control of excitation frequencies. 

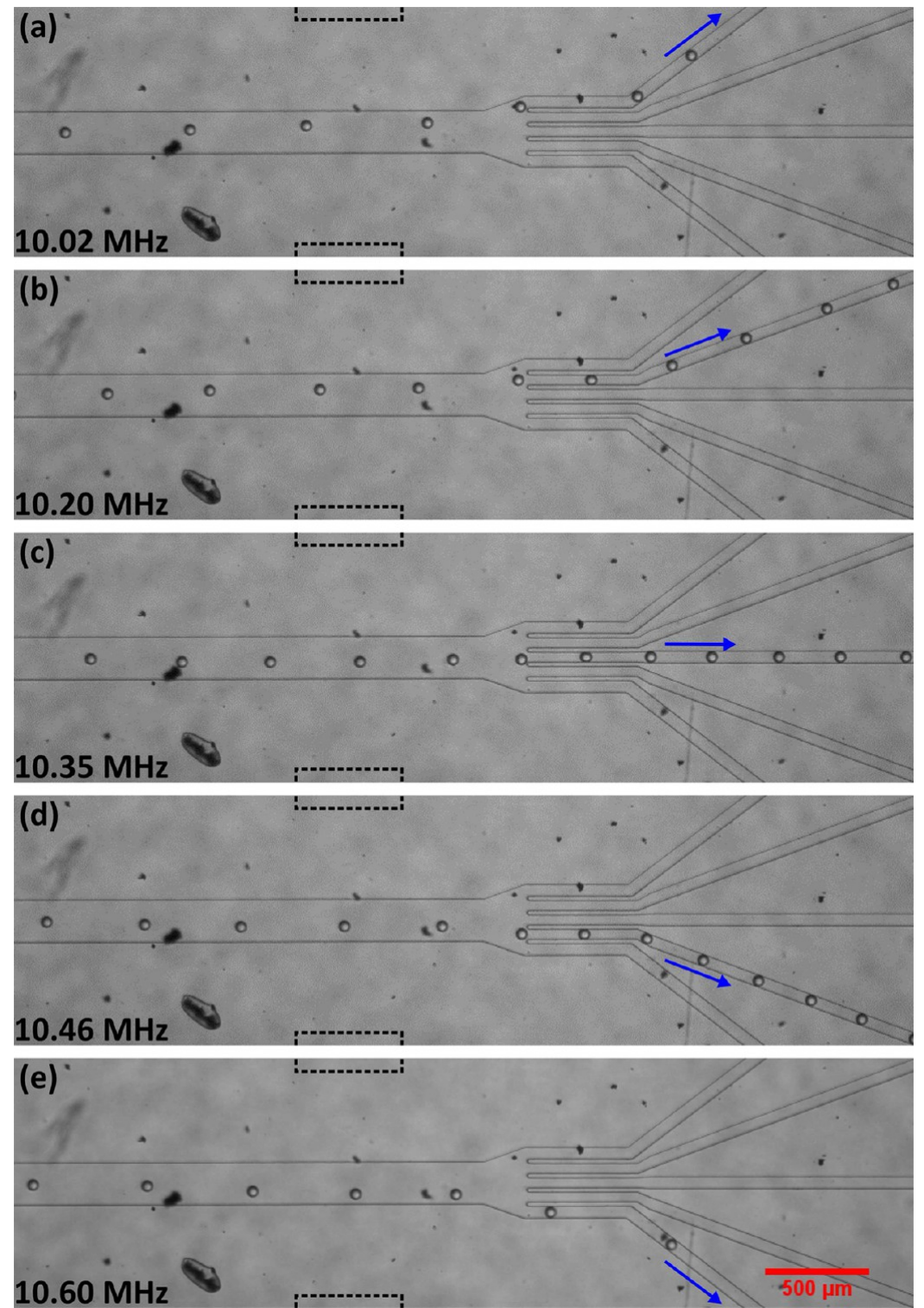

Figure 2. (a-e) Diagrams showing the directed droplet flows into five outlet channels under five different SSAW frequencies. The blue arrow indexes the target outlet, and the dashed lines indicate the aperture position of the chirped IDTs relative to the microchannel.

\section{EXPERIMENTS}

Device Fabrication. A photographic image of our device is shown as Figure $1 \mathrm{~b}$. The fabrication procedure for the SSAWbased droplet-sorting device includes: (1) the fabrication of a SAW substrate, (2) the fabrication of a PDMS microchannel, (3) the bonding of the PDMS microchannel to the SAW substrate. To fabricate the SAW substrate, a double layer of chrome and gold $(\mathrm{Cr} / \mathrm{Au}, 50 \AA / 500 \AA)$ was deposited on a photoresist-patterned $128^{\circ}$ Y-cut lithium niobate $\left(\mathrm{LiNbO}_{3}\right)$ wafer ( $500 \mu \mathrm{m}$ thick, double-side polished), followed by a liftoff technique to form the pair of chirped IDTs. The chirped IDTs in our experiment have 27 pairs of electrodes with the width of electrode and spacing gap increasing linearly from 90 to $100 \mu \mathrm{m}$ by an increment of $1.25 \mu \mathrm{m}$, each width repeating three pairs. The PDMS microchannel was fabricated by standard soft-lithography using SU-8 photoresist. After holes were drilled on the PDMS microchannel for inlets and outlets with a Harris Uni-Core 1.0-mm punch, the PDMS microchannel was treated with oxygen plasma in a plasma cleaner
(Harrick Plasma) for $3 \mathrm{~min}$ with the SAW substrate. The PDMS microchannel was then aligned and bonded on the SAW substrate in between the chirped IDTs after which the whole device was cured at $65{ }^{\circ} \mathrm{C}$ for at least three days before experiments to ensure that the surfaces of the microchannel were hydrophobic.

System Setup. All the experiments were conducted on the stage of an inverted microscope (Nikon Eclipse Ti-U). In order to eliminate the virtual image introduced by using a double-side polished $\mathrm{LiNbO}_{3}$ substrate, we placed a polarizer in the light path and adjusted it to a certain angle. For droplet generation, Fluorinert FC-40 (Sigma) with 3 wt \% DuPont Krytox 157 FS (ChemPoint) was used as continuous oil phase while deionized water was used as the dispersed water phase. Fluid prepared in 1-mL plastic syringes (Becton, Dickinson and Company) was injected through polyethylene tubing (Becton, Dickinson and Company) into the microchannel using neMESYS syringe pumps (cetoni $\mathrm{GmbH}$, Germany). An RF signal function generator (Agilent E4422B) was used to generate RF signals at desired frequencies, which were applied to the chirped IDTs 
after amplification by a power amplifier (Amplifier Research $100 \mathrm{~A} 250 \mathrm{~A})$ to generate the SSAW field.

Characterization and Data Analysis. A fast camera (Photron SA4, Japan) was connected to the microscope for data acquisition at a speed of 2000 frames per second (fps) during the experiment. Recorded data was analyzed with Image J (NIH, Bethesda, MD, U.S.A.). In Figure 3, the droplet position was traced by analyzing the gray value of the stacked image of 500 frames and plotted in MATLAB. In Figure 5a, the droplet generation was analyzed from the captured video with the "Plot $Z$-axis Profile" function and plotted in MATLAB. In Figure $5 b$, the input pulsed signal after amplification was recorded with a digital phosphor oscilloscope (load set at 1 $\mathrm{M} \Omega$ ) (Tektronix DPO4104) and plotted in MATLAB.

\section{RESULTS AND DISCUSSION}

SSAW-Based Droplet Switching. We first tested the device's ability to switch droplet streams to each of the five outlet channels. Micrographs indicating directed droplet flows into five outlet channels under five different SSAW frequencies are shown in Figure 2. In this experiment, a narrow chirped IDTs device with aperture of $500 \mu \mathrm{m}$ was used. The flow rates of the water-phase inlet and two oil-phase inlets were 0.2 and $8.0 \mu \mathrm{L} / \mathrm{min}$, respectively; no oil sheath flow was used. With a $40 \mu \mathrm{m} \times 50 \mu \mathrm{m}$ cross section of the flow-focusing nozzle, droplets with diameter of $\sim 50 \mu \mathrm{m}$ were generated under these flow rates. At the beginning of the experiment we first tuned the input SSAW frequency to identify five working frequencies $\left(f_{1}=10.02 \mathrm{MHz}, f_{2}=10.20 \mathrm{MHz}, f_{3}=10.35 \mathrm{MHz}, f_{4}=10.46\right.$ $\mathrm{MHz}$, and $f_{5}=10.60 \mathrm{MHz}$ ) under which droplets could be directed into five outlet channels when the SSAW was excited. Then, by applying an RF signal periodically sweeping among these five working frequencies with a mean peak-to-peak voltage of around $48.0 \mathrm{~V}$ to the chirped IDTs, we were able to switch droplet flows among the five outlets, as shown in Figure $2 \mathrm{a}-\mathrm{e}$. Video 1 shows these results and is available in the Supporting Information (SI). In our future work, a flowfocusing nozzle with a smaller cross section will be used to generate droplets with smaller diameters (e.g., $30 \mu \mathrm{m}$ ). With this improvement, it is possible to further increase the number of outlets (e.g., seven or more) in which droplets can be sorted in one step.

The effect of input voltage on the lateral displacement of droplets across the microchannel was then investigated for two different SSAW frequencies (10.02 and $10.20 \mathrm{MHz}$ ) using the same device under the same flow conditions. The RF signal was applied to the chirped IDTs with four different mean peak-topeak voltages $(30.0,38.4,48.0$ and $60.0 \mathrm{~V})$, and the position of droplets within the last $2400 \mu \mathrm{m}$ of the main channel at each voltage was recorded and plotted, as shown in Figure 3. When the chirped IDTs were excited, higher input voltage generated stronger SSAW field with larger acoustic radiation force, which pushed droplets faster in the $y$-direction. This indicated that the velocity of droplet in the $y$-direction increased with the input voltage. Under the excitation of the $10.02 \mathrm{MHz}$ RF signal, since the pressure node (the node nearest to the droplet stream) was located far from the centerline of the microchannel, the droplets never reached the pressure node and were still migrating in the $y$-direction as they left the SSAW field. Because the flow rates were constant, each droplet traveled through the SSAW field in the $x$-direction for the same amount of time regardless of the voltage. Therefore, the lateral displacement of droplets increased with the voltage (Figure 3a). As a result, the
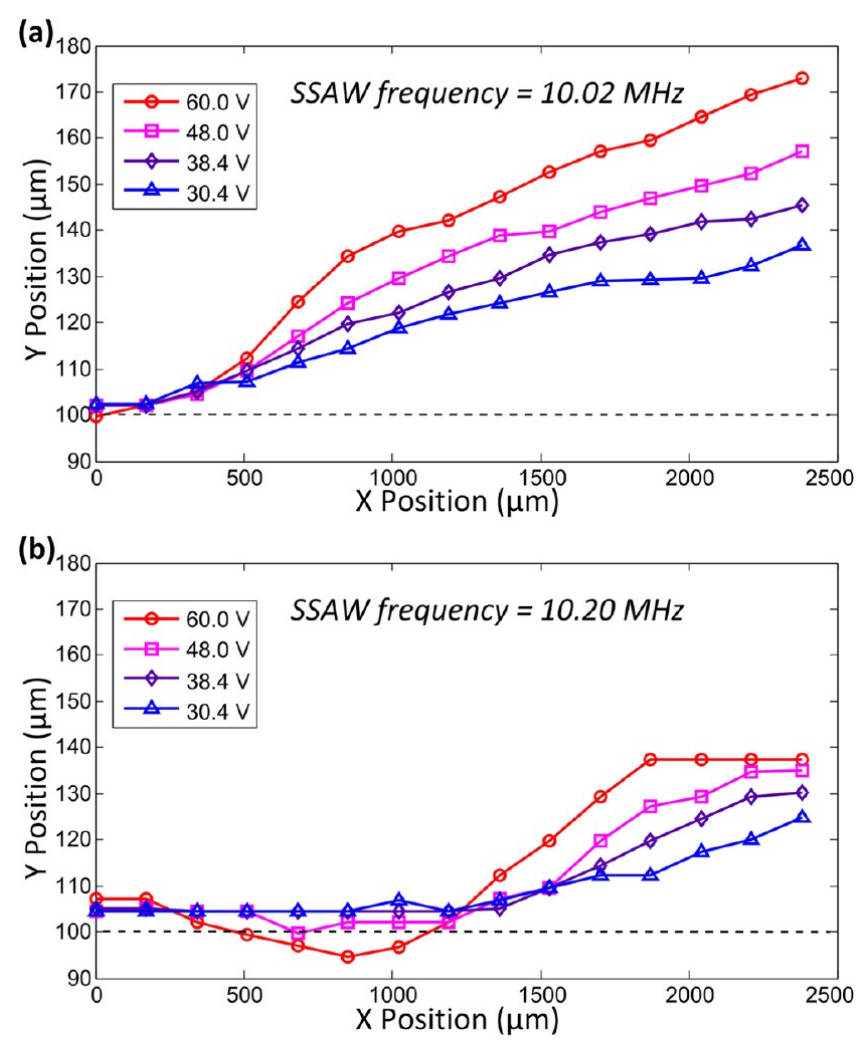

Figure 3. The relationship between droplet lateral displacement in the $y$-direction and droplet position along the channel in the $x$-direction at different voltages under two SSAW frequencies, (a) $10.02 \mathrm{MHz}$ and (b) $10.20 \mathrm{MHz}$. Black dashed line indicates the centerline of the microchannel. The origin of the coordinates was set at the lower channel wall $2400 \mu \mathrm{m}$ away from the outlet region with the $x$-axis along the lower channel wall and the $y$-axis normal to the channel wall.

sorting outcome was also influenced: droplets exited through the uppermost outlet at 60.0 and $48.0 \mathrm{~V}$ while through the second uppermost outlet at 38.0 and $30.4 \mathrm{~V}$ (data not shown). In contrast, under the excitation of the $10.20 \mathrm{MHz}$ RF signal, the pressure node was shifted nearer to the centerline of the microchannel. Hence, droplets would have reached the pressure node before leaving the SSAW field above certain voltage. As shown by the red curve in Figure $3 \mathrm{~b}(60.0 \mathrm{~V})$, the lateral displacement of droplets did not increase after $X=1870 \mu \mathrm{m}$ because droplets had already reached the pressure node. In this circumstance, droplets could never flow into the uppermost outlet regardless of the input voltage.

SSAW-Based Single Droplet Sorting. In order to develop a fluorescence-activated droplet sorter (FADS), the ability to sort a single droplet out of a stream is desirable. Therefore, we tested the feasibility of single droplet sorting by applying a pulsed RF signal to the narrow chirped IDTs. Micrographs demonstrating the diversion of single droplets from the main stream into four different target outlets are shown in Figure 4. In this experiment, the same chirped IDTs device with aperture of $500 \mu \mathrm{m}$ was used. When the SSAW was off, all the droplets flowed into the center outlet channel by default. When a pulsed signal of desired frequency with certain pulse width was applied, one could deflect a single droplet into the desired target outlet channel. In (b) and (c) of Figure 4, the flow rates of the water-phase inlet, two oil-phase inlets and two sheathflow inlets were $0.2,8.0$, and $1.7 \mu \mathrm{L} / \mathrm{min}$, respectively. By applying a $15 \mathrm{~ms}$ wide pulsed signal of 10.20 or $10.45 \mathrm{MHz}$ 


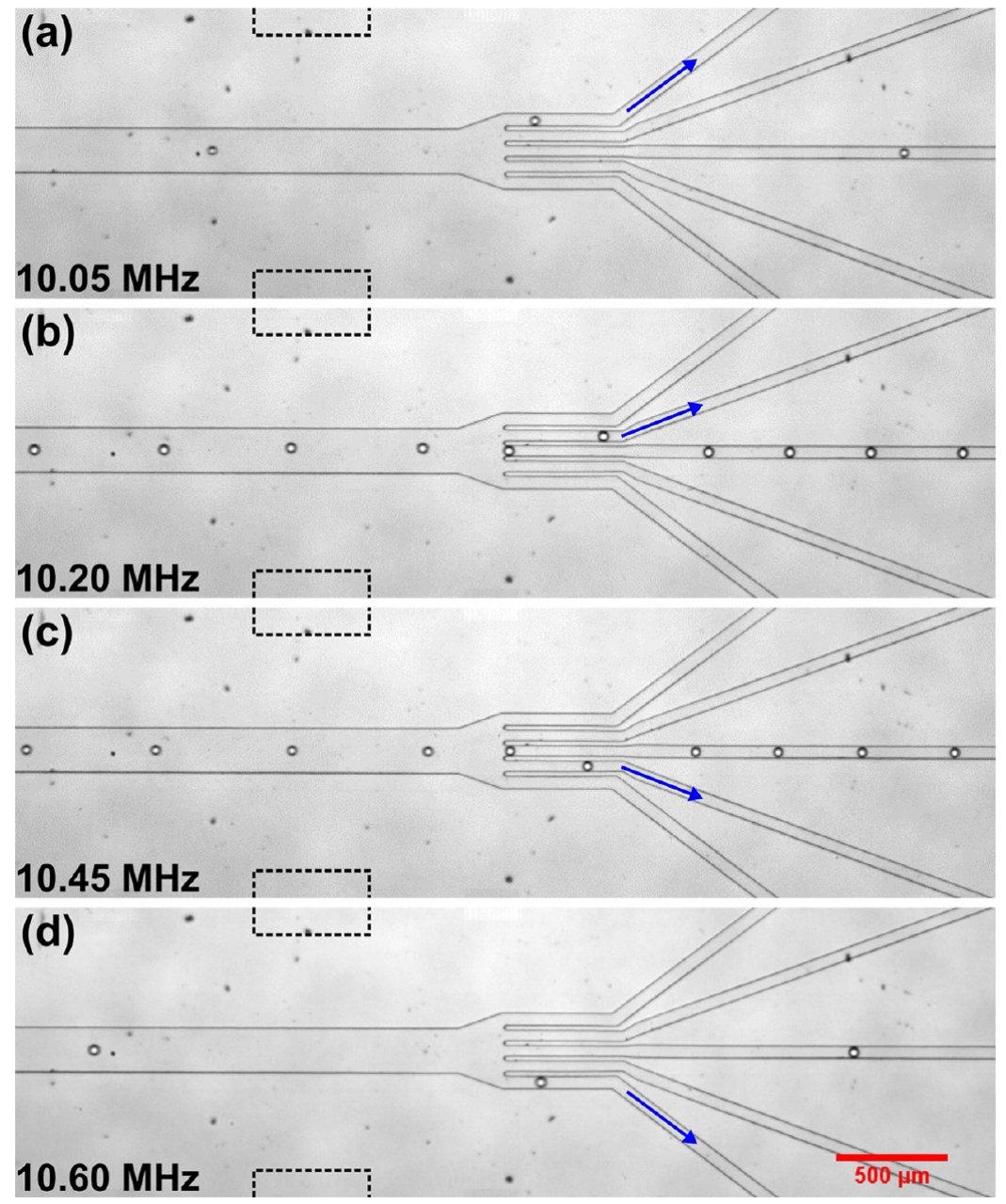

Figure 4. $(\mathrm{a}-\mathrm{d})$ show the sorting of single droplets into four different target outlets under pulsed RF signal. The dashed lines indicate the aperture position of the chirped IDTs relative to the microchannel.

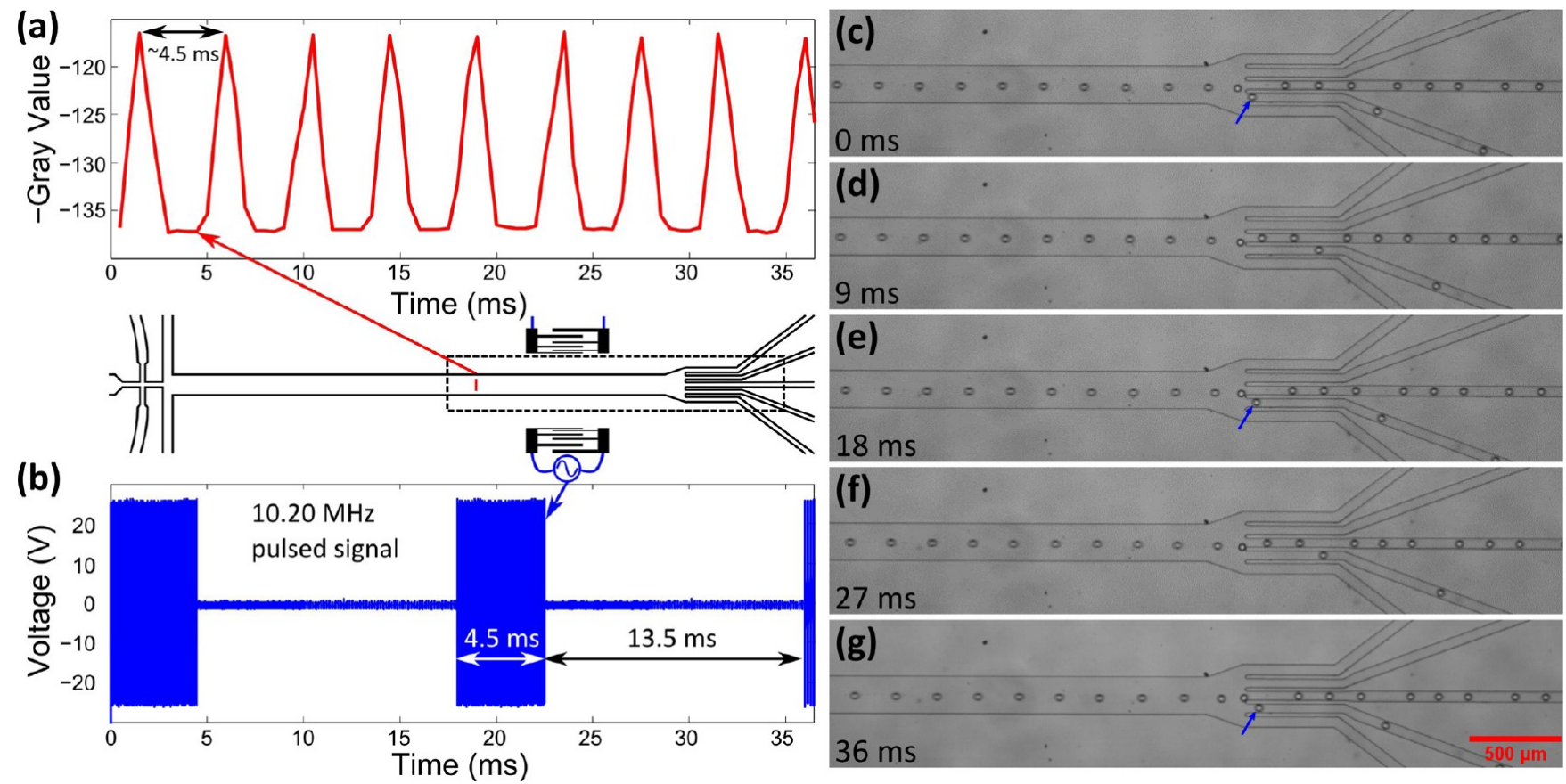

Figure 5. Sorting of single droplets at rate of 222 droplets s $^{-1}$. (a) shows the droplet generation, (b) shows the $10.20 \mathrm{MHz}$ input pulsed signal, and $(\mathrm{c}-\mathrm{g})$ show the sorting of three single droplets out of the stream (indicated by blue arrows). 
with mean peak-to-peak voltage of $48.0 \mathrm{~V}$, we successfully diverted a single droplet either upward or downward to the neighboring target outlet. In comparison, the sorting of a single droplet into the uppermost or lowermost target outlet is more difficult, since a larger lateral displacement of the droplet is required. To realize this, a longer pulse width and higher input voltage could help based on our discussion above. In (a) and (d) of Figure 4, the flow rates of the water-phase inlet, two oilphase inlets and two sheath-flow inlets were 0.1, 8.0, and 3.0 $\mu \mathrm{L} / \mathrm{min}$, respectively. Under this condition, we applied a $35 \mathrm{~ms}$ wide pulsed signal of 10.05 or $10.60 \mathrm{MHz}$ with mean peak-topeak voltage of $54.0 \mathrm{~V}$ to sort a single droplet into the uppermost or lowermost target outlet. Four video clips showing these results are available online in videos $2-5$ in the SI.

At last, we evaluated the throughput of our SSAW-based droplet sorter by using the chirped IDTs with smaller aperture $(300 \mu \mathrm{m})$, which gave us a better chance to sort a single droplet out from its neighboring droplets. These results are shown in Figure 5. In this experiment, droplets (diameter $\sim 40 \mu \mathrm{m}$ ) were generated roughly every $4.5 \mathrm{~ms}$, corresponding to a droplet generation rate of 222 droplets $\mathrm{s}^{-1}$ (Figure 5a). In order to sort single droplets out of the stream, we applied a $10.20 \mathrm{MHz}$ periodically oscillating pulsed signal with a pulse width of 4.5 ms and pulse period of $18 \mathrm{~ms}$ to the chirped IDTs (duty cycle is $25 \%$ ) (Figure $5 \mathrm{~b}$ ). As a consequence, a single droplet was sorted into the target outlet about every $18 \mathrm{~ms}$, as shown in diagrams $\mathrm{c}-\mathrm{g}$ of Figure 5. Video 6 shows these results and is available in the SI. The sorting performance of our SSAWbased approach is promising since whenever the SSAW was on during the pulse of $4.5 \mathrm{~ms}$, one droplet was sorted out. Therefore, we have every reason to believe that with the integration of an optical-detection unit to detect the target droplet and a trigger-control unit to control the SSAW generation, we can better match the SSAW generation with the target droplet to achieve single droplet sorting with high accuracy. To further increase the throughput in our future work, there are several possible approaches. The first approach is to fabricate chirped IDTs with an even smaller aperture (e.g., $100 \mu \mathrm{m})$ and more pairs of electrodes. This modified design can help to generate a narrower SSAW field while reducing the effect of acoustic dispersion at the edge. Thus, it can help to sort single droplets with closer interdroplet distances at higher throughput. The second approach is to apply a pulsed signal with a higher voltage. Under higher voltages, the acoustic radiation force is larger, which pushes the droplet at a higher velocity in the $y$-direction. Therefore, a shorter time or pulse width (and thus less translational distance of the droplet in the $x$-direction) will be needed to sort the single droplet into a certain target outlet. This can also lead to higher-throughput single droplet sorting. The third approach to increase the sorting throughput is to decrease the distance between the two IDTs and bond the microchannel closer to the IDTs. This can also reduce the effect of acoustic dispersion to increase the sorting throughput.

\section{CONCLUSIONS}

In summary, we have successfully developed and tested a SSAW-based multichannel droplet sorter. Our device integrates on-chip, water-in-oil droplet formation with SSAW-based, multichannel droplet sorting. Our device takes advantages of the excellent controllability and tunability offered by chirped IDTs and can sort picoliter droplets into five (or more) outputs at rates up to 222 droplets $\mathrm{s}^{-1}$ without external labeling. With further optimization, this device can be integrated with an optical-detection module and other on-chip functions to enable a SSAW-based, micro fluorescence-activated droplet sorter $(\mu \mathrm{FADS})$.

\section{ASSOCIATED CONTENT}

S Supporting Information

Videos 1-6. This material is available free of charge via the Internet at http://pubs.acs.org.

\section{AUTHOR INFORMATION}

\section{Corresponding Author}

*Fax: 814-865-9974. Tel: 814-863-4209. E-mail: junhuang@ psu.edu.

\section{Notes}

The authors declare no competing financial interest.

\section{ACKNOWLEDGMENTS}

We thank Joey Rufo for the help in preparing the manuscript and ChemPoint for providing DuPont Krytox 157 FS surfactant. This research was supported by the National Institutes of Health (NIH) Director's New Innovator Award (1DP2OD007209-01), the National Science Foundation, and the Penn State Center for Nanoscale Science (MRSEC) under Grant DMR-0820404. S.L. and C.E.C. were funded in part by grant AI45818 from NIAID, NIH. Components of this work were conducted at the Penn State node of the NSF-funded National Nanotechnology Infrastructure Network (NNIN).

\section{REFERENCES}

(1) Thorsen, T.; Roberts, R. W.; Arnold, F. H.; Quake, S. R. Phys. Rev. Lett. 2001, 86, 4163.

(2) Anna, S. L.; Bontoux, N.; Stone, H. A. Appl. Phys. Lett. 2003, 82, 364.

(3) Guo, F.; Lapsley, M. I.; Nawaz, A. A.; Zhao, Y.; Lin, S.-C. S.; Chen, Y.; Yang, S.; Zhao, X.-Z.; Huang, T. J. Anal. Chem. 2012, 84, 10745 .

(4) Huebner, A.; Sharma, S.; Srisa-Art, M.; Hollfelder, F.; Edel, J. B.; deMello, A. J. Lab Chip 2008, 8, 1244.

(5) Guo, M. T.; Rotem, A.; Heyman, J. A.; Weitz, D. A. Lab Chip 2012, 12, 2146.

(6) Theberge, A. B.; Courtois, F.; Schaerli, Y.; Fischlechner, M.; Abell, C.; Hollfelder, F.; Huck, W. T. S. Angew. Chem., Int. Ed. 2010, 49, 5846.

(7) Griffiths, A. D.; Tawfik, D. S. Trends Biotechnol. 2006, 24, 395.

(8) Song, H.; Chen, D. L.; Ismagilov, R. F. Angew. Chem., Int. Ed. 2006, 45, 7336 .

(9) Clausell-Tormos, J.; Lieber, D.; Baret, J.-C.; El-Harrak, A.; Miller, O. J.; Frenz, L.; Blouwolff, J.; Humphry, K. J.; Köster, S.; Duan, H.; Holtze, C.; Weitz, D. A.; Griffiths, A. D.; Merten, C. A. Chem. Biol. 2008, 15, 427.

(10) Fallah-Araghi, A.; Baret, J.-C.; Ryckelynck, M.; Griffiths, A. D. Lab Chip 2012, 12, 882.

(11) Miller, O. J.; El-Harrak, A.; Mangeat, T.; Baret, J.-C.; Frenz, L.; El Debs, B.; Mayot, E.; Samuels, M. L.; Rooney, E. K.; Dieu, P.; Galvan, M.; Link, D. R.; Griffiths, A. D. Proc. Natl. Acad. Sci. U.S.A. 2012, 109, 378.

(12) Neuži, P.; Giselbrecht, S.; Länge, K.; Huang, T. J.; Manz, A. Nat. Rev. Drug Discovery 2012, 11, 620.

(13) Schaerli, Y.; Wootton, R. C.; Robinson, T.; Stein, V.; Dunsby, C.; Neil, M. A. A.; French, P. M. W.; deMello, A. J.; Abell, C.; Hollfelder, F. Anal. Chem. 2009, 81, 302.

(14) Srisa-Art, M.; deMello, A. J.; Edel, J. B. Anal. Chem. 2007, 79, 6682.

(15) Mao, X.; Huang, T. J. Lab Chip 2012, 12, 1412. 
(16) Chabert, M.; Viovy, J.-L. Proc. Natl. Acad. Sci. U.S.A. 2008, 105, 3191

(17) Joensson, H. N.; Uhlén, M.; Svahn, H. A. Lab Chip 2011, 11, 1305.

(18) Zhang, K.; Liang, Q.; Ai, X.; Hu, P.; Wang, Y.; Luo, G. Lab Chip 2011, 11, 1271.

(19) Shemesh, J.; Bransky, A.; Khoury, M.; Levenberg, S. Biomed. Microdevices 2010, 12, 907.

(20) Robert de Saint Vincent, M.; Wunenburger, R.; Delville, J.-P. Appl. Phys. Lett. 2008, 92, 154105.

(21) Niu, X.; Zhang, M.; Wu, J.; Wen, W.; Sheng, P. Soft Matter 2009, 5, 576.

(22) Niu, X.; Zhang, M.; Peng, S.; Wen, W.; Sheng, P. Biomicrofluidics 2007, 1, 44101.

(23) Link, D. R.; Grasland-Mongrain, E.; Duri, A.; Sarrazin, F.; Cheng, Z.; Cristobal, G.; Marquez, M.; Weitz, D. A. Angew. Chem., Int. Ed. 2006, 45, 2556.

(24) Lee, C.-Y.; Lin, Y.-H.; Lee, G.-B. Microfluid. Nanofluid. 2008, 6, 599.

(25) Guo, F.; Ji, X.-H.; Liu, K.; He, R.-X.; Zhao, L.-B.; Guo, Z.-X.; Liu, W.; Guo, S.-S.; Zhao, X.-Z. Appl. Phys. Lett. 2010, 96, 193701.

(26) Baroud, C.; Delville, J.-P.; Gallaire, F.; Wunenburger, R. Phys. Rev. E 2007, 75, 1 .

(27) Baret, J.-C.; Miller, O. J.; Taly, V.; Ryckelynck, M.; El-Harrak, A.; Frenz, L.; Rick, C.; Samuels, M. L.; Hutchison, J. B.; Agresti, J. J.; Link, D. R.; Weitz, D. A.; Griffiths, A. D. Lab Chip 2009, 9, 1850.

(28) Ahn, K.; Kerbage, C.; Hunt, T. P.; Westervelt, R. M.; Link, D. R.; Weitz, D. A. Appl. Phys. Lett. 2006, 88, 024104.

(29) Ahn, B.; Lee, K.; Panchapakesan, R.; Oh, K. W. Biomicrofluidics 2011, 5, 24113.

(30) Ahn, B.; Lee, K.; Louge, R.; Oh, K. W. Biomicrofluidics 2009, 3, 44102.

(31) Abate, A. R.; Agresti, J. J.; Weitz, D. A. Appl. Phys. Lett. 2010, 96, 203509.

(32) Lee, C.; Lee, J.; Kim, H. H.; Teh, S.-Y.; Lee, A.; Chung, I.-Y.; Park, J.; Shung, K. K. Lab Chip 2012, 12, 2736.

(33) Franke, T.; Abate, A. R.; Weitz, D. A.; Wixforth, A. Lab Chip 2009, 9, 2625.

(34) Ahmed, D.; Mao, X.; Juluri, B. K.; Huang, T. J. Microfluid. Nanofluid. 2009, 7, 727.

(35) Ahmed, D.; Mao, X.; Shi, J.; Juluri, B. K.; Huang, T. J. Lab Chip 2009, 9, 2738.

(36) Alvarez, M.; Yeo, L. Y.; Friend, J. R.; Jamriska, M. Biomicrofluidics 2009, 3, 14102.

(37) Franke, T.; Braunmüller, S.; Schmid, L.; Wixforth, A.; Weitz, D. A. Lab Chip 2010, 10, 789.

(38) Li, H.; Friend, J. R.; Yeo, L. Y. Biomed. Microdevices 2007, 9, 647.

(39) Li, H.; Friend, J. R.; Yeo, L. Y. Phys. Rev. Lett. 2008, 101, 1.

(40) Lin, S.-C. S.; Mao, X.; Huang, T. J. Lab Chip 2012, 12, 2766.

(41) Liu, Y.; Lim, K.-M. Lab Chip 2011, 11, 3167.

(42) Meng, L.; Cai, F.; Chen, J.; Niu, L.; Li, Y.; Wu, J.; Zheng, H. Appl. Phys. Lett. 2012, 100, 173701.

(43) Meng, L.; Cai, F.; Jin, Q.; Niu, L.; Jiang, C.; Wang, Z.; Wu, J.; Zheng, H. Sens. Actuators, B 2011, 160, 1599.

(44) Meng, L.; Cai, F.; Zhang, Z.; Niu, L.; Jin, Q.; Yan, F.; Wu, J.; Wang, Z.; Zheng, H. Biomicrofluidics 2011, 5, 044104.

(45) Nam, J.; Lee, Y.; Shin, S. Microfluid. Nanofluid. 2011, 11, 317.

(46) Nam, J.; Lim, H.; Kim, C.; Kang, J. Y.; Shin, S. Biomicrofluidics 2012, 6, 024120.

(47) Chen, Y.; Ding, X.; Lin, S. S.-C.; Yang, S.; Huang, P.-H.; Nama, N.; Zhao, Y.; Nawaz, A. A.; Guo, F.; Wang, W.; Gu, Y.; Mallouk, T. E.; Huang, T. J. ACS Nano 2013, 7, 3306.

(48) Liu, Y. J.; Ding, X.; Lin, S. S.-C.; Shi, J.; Chiang, I.-K.; Huang, T. J. Adv. Mater. 2011, 23, 1656.

(49) Petersson, F.; Aberg, L.; Swärd-Nilsson, A.-M.; Laurell, T. Anal. Chem. 2007, 79, 5117.

(50) Petersson, F.; Nilsson, A.; Jönsson, H.; Laurell, T. Anal. Chem. 2005, 77, 1216.

(51) Qi, A.; Yeo, L. Y.; Friend, J. R.; Ho, J. Lab Chip 2010, 10, 470.
(52) Tan, M.; Friend, J. R.; Yeo, L. Y. Phys. Rev. Lett. 2009, 103, 1. (53) Wood, C. D.; Cunningham, J. E.; O’Rorke, R.; Wälti, C.; Linfield, E. H.; Davies, A. G.; Evans, S. D. Appl. Phys. Lett. 2009, 94, 054101 .

(54) Wood, C. D.; Evans, S. D.; Cunningham, J. E.; O'Rorke, R.; Wälti, C.; Davies, A. G. Appl. Phys. Lett. 2008, 92, 044104.

(55) Xie, Y.; Ahmed, D.; Lapsley, M. I.; Lin, S. S.; Nawaz, A. A.; Wang, L.; Huang, T. J. Anal. Chem. 2012, 84, 7495.

(56) Xie, Y.; Zhao, C.; Zhao, Y.; Li, S.; Rufo, J.; Yang, S.; Guo, F.; Huang, T. J. Lab Chip 2013, 13, 1772.

(57) Mao, X.; Huang, T. J. Lab Chip 2012, 12, 4006.

(58) Ahmed, D.; Chan, C. Y.; Lin, S.-C. S.; Muddana, H. S.; Nama, N.; Benkovic, S. J.; Huang, T. J. Lab Chip 2013, 13, 328.

(59) Lin, S.-C. S.; Mao, X.; Huang, T. J. Lab Chip 2012, 12, 2766.

(60) Shi, J.; Yazdi, S.; Lin, S.-C. S.; Ding, X.; Chiang, I.-K.; Sharp, K.; Huang, T. J. Lab Chip 2011, 11, 2319.

(61) Shi, J.; Mao, X.; Ahmed, D.; Colletti, A.; Huang, T. J. Lab Chip 2008, 8, 221.

(62) Shi, J.; Huang, H.; Stratton, Z.; Huang, Y.; Huang, T. J. Lab Chip 2009, 9, 3354.

(63) Shi, J.; Ahmed, D.; Mao, X.; Lin, S.-C. S.; Lawit, A.; Huang, T. J. Lab Chip 2009, 9, 2890.

(64) Ding, X.; Shi, J.; Lin, S.-C. S.; Yazdi, S.; Kiraly, B.; Huang, T. J. Lab Chip 2012, 12, 2491.

(65) Ding, X.; Lin, S.-C. S.; Lapsley, M. I.; Li, S.; Guo, X.; Chan, C. Y.; Chiang, I.-K.; Wang, L.; McCoy, J. P.; Huang, T. J. Lab Chip 2012, $12,4228$.

(66) Ding, X.; Lin, S.-C. S.; Kiraly, B.; Yue, H.; Li, S.; Chiang, I.; Shi, J.; Benkovic, S. J.; Huang, T. J. Proc. Natl. Acad. Sci. U.S.A. 2012, 109, 11105 .

(67) Arora, A.; Simone, G.; Salieb-Beugelaar, G. B.; Kim, J. T.; Manz, A. Anal. Chem. 2010, 82, 4830.

(68) Wheeler, A. R.; Throndset, W. R.; Whelan, R. J.; Leach, A. M.; Zare, R. N.; Liao, Y. H.; Farrell, K.; Manger, I. D.; Daridon, A. Anal. Chem. 2003, 75, 3581.

(69) Ng, A. H. C.; Choi, K.; Luoma, R. P.; Robinson, J. M.; Wheeler, A. R. Anal. Chem. 2012, 84, 8805.

(70) Pamme, N.; Manz, A. Anal. Chem. 2004, 76, 7250.

(71) Riahi, R.; Mach, K. E.; Mohan, R.; Liao, J. C.; Wong, P. K. Anal. Chem. 2011, 83, 6349.

(72) Sin, M. L. Y.; Liu, T.; Pyne, J. D.; Gau, V.; Liao, J. C.; Wong, P. K. Anal. Chem. 2012, 84, 2702.

(73) Hughes, A. J.; Lin, R. K. C.; Peehl, D. M.; Herr, A. E. Proc. Natl. Acad. Sci. U.S.A. 2012, 109, 5972.

(74) Karns, K.; Herr, A. E. Anal. Chem. 2011, 83, 8115.

(75) Xia, Y.; Whitesides, G. M. Annu. Rev. Mater. Sci. 1998, 28, 153.

(76) Yosioka, K.; Kawasima, Y. Acustica 1955, 5, 167. 